\title{
SuperMassive Blackholes grow from stellar BHs of star formation history?
}

\author{
Brigitte Rocca-Volmerange \\ CNRS-Institut d'Astrophysique de Paris \\ 98bis Bd Arago, 75014 Paris, France (and Université Paris Sud) \\ email: rocca@iap.fr
}

\begin{abstract}
The origin of the supermassive black hole masses $\mathrm{M}_{S M B H}$ discovered at the highest redshifts is still actively debated. Moreover the statistically significant relation of $\mathrm{M}_{S M B H}$ with bulge luminosities $\mathrm{L}_{V}$, extended on several magnitude orders, confirms a common physical process linking small $(\leqslant 1 \mathrm{pc})$ to large $(\mathrm{kpcs})$ size scales. The Spectral Energy Distributions (SEDs) of two $z=3.8$ radio galaxies $4 \mathrm{C} 41.17$ and TN J2007-1316, best-fitted by evolved early type galaxy and starburst scenarios also imply masses of stellar remnants. Computed with the evolutionary code Pegase.3, the cumulated stellar black hole mass $\mathrm{M}_{s B H}$ reach up to several $10^{9} \mathrm{M}_{\odot}$, similar to $\mathrm{M}_{S M B H}$ at same z. We propose the $S M B H$ growth is due to the migration of the stellar dense residues $(\mathrm{sBH})$ towards the galaxy core by dynamical friction. Discussed in terms of time-scales, this process which is linking AGN and star formation, also fully justifies the famous relation $\mathrm{M}_{S M B H}-L_{V}$.
\end{abstract}

Keywords. stars: formation, supernovae, galaxies: evolution, high-redshift galaxies, nuclei, ISM: supernova remnants

\section{From the SED best-fits of $\mathrm{z}=3.8$ radio galaxies}

The two $\mathrm{z}=3.8$ radio galaxies $4 \mathrm{C} 41.17$ and $\mathrm{TN} \mathrm{J} 2007-1316$ are selected for their low AGN contribution from the distant radio galaxy sample HeRGE (Drouart et al., 2014). Their continuous multiwavelength SEDs from the HST, Spitzer and Herschel satellites (Seymour et al., 2007) are corrected for coherent apertures and calibrations. From RoccaVolmerange et al. 2013, the UV-visible to far-infrared star emissions (through stellar photospheres and dust) are modeled with the help of the evolutionary code Pegase.3 (Fioc, Rocca-Volmerange et al., near submission) by building a large number of synthetic libraries. The scenarios of Hubble type galaxies are robust, fitting the local SDSS colorcolor diagram and predicting significant photometric redshifts (Le Borgne \& RoccaVolmerange, 2002) or faint galaxy counts. Fig. 1 shows that the starburst age of 30 Myrs reproduces the far-IR emission while the old component is needed in the near-IR and the optical. At 30 Myrs, all supernovae of lifetime duration $\leqslant 30 \mathrm{Myrs}$ already exploded into dense residues (stellar black hole or neutron stars) and ejecta enriching the ISM. By subtracting the ejecta mass from each supernova mass, its dense residue mass is derived. The code Pegase. 3 estimates the cumulated mass $\mathrm{M}_{s B H}$ of dense residues to a few $10^{9} M_{\odot}$ comparable to $M_{S M B H}$ measured at similar redshifts (Rocca-Volmerange et al., 2015 ).

\section{The migration process}

According to Binney \& Tremaine, 2008, the timescale $\mathrm{t}_{\text {fric }}$ for a compact object to decay towards the galactic center through dynamical friction might be long in case of a standard $10 \mathrm{M}_{\odot}$ stellar black hole at the scale of a few kpcs of a radio galaxy disk. Both 


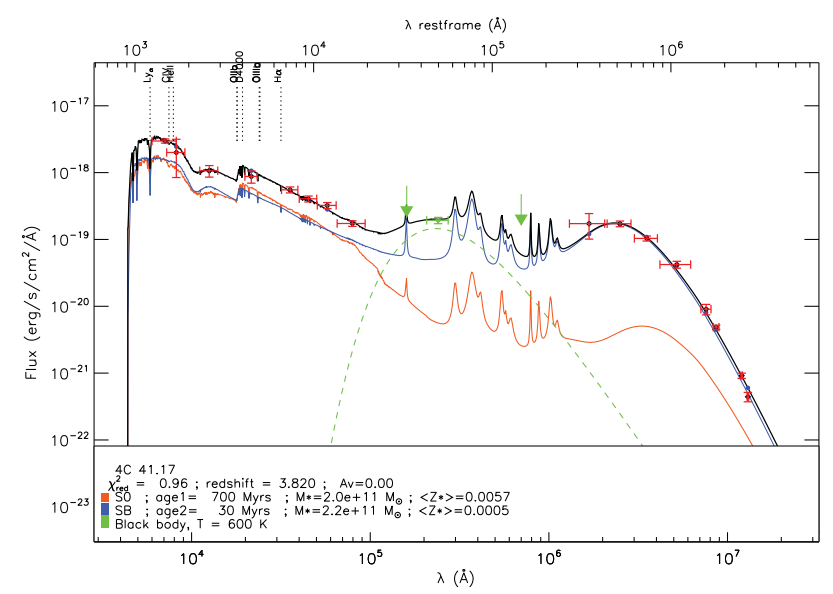

Figure 1. Best-fit (black) of the continuous SED observations (red + ) of the $z=3.8$ radio galaxy 4C41.17. Components are: old S0 galaxy (orange), evolved starburst (blue) and AGN thermal emission (green dashed). Green points and arrows are not fit-included.

Table 1. Ages, luminous star mass $\mathrm{M}_{\text {star }}$, stellar Black Hole mass $\mathrm{M}_{s B H}$ and metallicity $\mathrm{Z}$ of the young (SB) and early (Elliptical/S0) components of the two $\mathrm{z}=3.8$ radio galaxies.

\begin{tabular}{|l|c|c|c|c|}
\hline $\begin{array}{l}\text { Galaxy } \\
\text { Comp. }\end{array}$ & $\begin{array}{c}\text { Age } \\
{[\mathrm{Myrs}]}\end{array}$ & $\begin{array}{c}\mathbf{M}_{\text {star }} \\
{\left[10^{11} M_{\odot}\right]}\end{array}$ & $\begin{array}{c}\mathbf{M}_{S B H} \\
{\left[10^{9} M_{\odot}\right]}\end{array}$ & $\mathbf{Z}$ \\
\hline 4 C41.17SB & 30 & 2.2 & 8.5 & $|0.0005|$ \\
\hline 4 C41.17S0 & 700 & 2.0 & 4.3 & $0.0057 \mid$ \\
\hline TN J2007-1316SB & 35 & 0.8 & 3.4 & $|0.0001|$ \\
\hline TN J2007-1316Ell & 1200 & 9.4 & 19.0 & $\mid 0.0155$ \\
\hline
\end{tabular}

Spitzer-Herschel SEDs and SLOAN data correspond to apertures $\geqslant 5$ arcsecs. Higher spatial resolution images of 4C41.17 from HST (Miley et al. 1992) and VLA (Carilli et al. 1994) give star formation zones of $\leqslant 250 p c$ and star masses $\left(10^{10} M_{\odot}\right)$ inducing $\mathrm{M}_{s B H}$ mass of $\simeq 10^{7} \mathrm{M}_{\odot}$ with the final $\mathrm{t}_{\text {fric }} \approx 0.1 \mathrm{Gyr}$ respecting the cosmic time of 1.6 Gyr at $\mathrm{z}=3.8$ (Rocca-Volmerange et al., 2015). Probing the stellar black hole accretion for fueling central black holes is a simple explanation for SMBH growth, which corresponds to a similar star formation law for luminous stars and $s B H$. Moreover it would be the key of the known $\mathrm{M}_{S M B H}-L_{V}$ relation. More improvements of this approach is waited for by disentangling the AGN and cold grain emissions of more active radio galaxies from the distant HeRGE sample and the $\mathrm{z} \simeq 13 \mathrm{CRR}$ catalog, at present in progress.

\section{References}

Carilli, C. L., Owen, F. N., \& Harris, D. E., 1994, Astrophys. J., 107, 480

Drouart, G., De Breuck, C., Vernet, J., Seymour, N. et al., 2014, Astron. $\mathcal{E}$ Astrophys., 566, 53 Le Borgne, D., \& Rocca-Volmerange, B., 2002, Astron. ES Astrophys., 386, 446

Miley, G. K., Chambers, K. C., van Breugel, W. J. M.., et al., 1992, Astrophys. J., 401, L69

Rocca-Volmerange, B., Drouart, G., \& De Breuck, C., 2015, Astrophys. J. , 803, L8

Rocca-Volmerange, B., Drouart, G., De Breuck, C. et al., 2013, MNRAS, 429, 2780

Seymour, N., Stern, D., De Breuck, C., et al., 2007, Astrophys. J. Supp.Series, 171, 353 